\title{
Acute lymphoblastic leukemia following temozolomide treatment in a patient with glioblastoma: A case report and review of the literature
}

\author{
PENGFEI LIU ${ }^{1-3}$, PEIWEN LI ${ }^{1}$, TING LEI ${ }^{1}$, LIMEI QU ${ }^{4}$, HAIYAN HUANG $^{1}$ and QINGCHUN MU $^{2}$ \\ ${ }^{1}$ Department of Neurosurgery The First Hospital of Jilin University, Changchun, Jilin 130000; ${ }^{2}$ Department of Neurosurgery, \\ Hongqi Hospital of Mudanjiang Medical University, Mudanjiang, Heilongjiang 157011; ${ }^{3}$ Department of Neurosurgery, \\ Guangdong 999 Brain Hospital, Guangzhou, Guangdong 510510; ${ }^{4}$ Department of Pathology, \\ The First Hospital of Jilin University, Changchun, Jilin 130000, P.R. China
}

Received July 9, 2015; Accepted September 22, 2016

DOI: $10.3892 / \mathrm{ol} .2018 .8422$

\begin{abstract}
Temozolomide (TMZ) is a second-generation oral alkylating agent that functions against a number of central nervous system neoplasms, and is generally used to treat high-grade gliomas, including anaplastic astrocytoma and glioblastoma multiforme. Therapy-related secondary myelodysplastic syndrome and acute myeloid leukemia have been reported in patients following prolonged exposure to TMZ. However, TMZ-related acute lymphoblastic leukemia (ALL) is extremely rare. The present study describes the case of an 11-year-old boy with a 3-day history of generalized tonic-clonic seizures and a contrast-enhanced lesion in the left temporooccipital region with focal cystic degeneration, as detected by magnetic resonance imaging. The patient underwent craniotomy and gross-total resection andpathological analysis confirmed the diagnosis of giant cell glioblastoma. Postoperatively, the patient received TMZ-based concurrent chemoradiation during radiotherapy, and developed B-cell ALL 6 months following TMZ treatment. A thorough literature search identified only six published cases of TMZ-related ALL. The chemotherapeutic efficacy of TMZ has been identified, however, its leukemogenic potential should be emphasized among practitioners and patients. Further studies are required to determine the specific pathogenic mechanism of TMZ-related ALL. Close hematological monitoring of patients following TMZ treatment is vital and a high index of suspicion is necessary.
\end{abstract}

Correspondence to: $\mathrm{Dr}$ Qingchun $\mathrm{Mu}$, Department of Neurosurgery, Hongqi Hospital of Mudanjiang Medical University, 5 Tongxiang Road, Mudanjiang, Heilongjiang 157011, P.R. China E-mail:muqc@qq.com

Dr Haiyan Huang, Department of Neurosurgery, The First Hospital of Jilin University, 71 Xinmin Street, Changchun, Jilin 130000, P.R. China E-mail: huanghy@jlu.edu.cn

Key words: temozolomide, acute lymphoblastic leukemia, glioblastoma multiforme

\section{Introduction}

Temozolomide (TMZ) is a second-generation oral alkylating agent with the ability to penetrate the blood-brain barrier, and is widely used in the management of high-grade brain neoplasms, including anaplastic astrocytoma and glioblastoma multiforme, in addition to brain metastasis from solid tumors $(1,2)$. TMZ is a prodrug and imidazotetrazine analog that exerts its action following spontaneous conversion to its active form, 5-(3-methyltriazen-1-yl)imidazole-4-carboxamide (3). This active drug subsequently exerts its antitumor effect by methylating the purine bases of chromosomal DNA including O6-guanine, which results in the failure of DNA replication, cell cycle arrest and subsequent apoptosis $(4,5)$. TMZ is generally considered effective and relatively safe (6-8); however, increased survival rates in certain patients have uncovered toxicities arising from the long-term use of alkylating agents, including TMZ (9). Treatment-related secondary myelodysplastic syndrome (t-MDS) and treatment-related acute myeloid leukemia (t-AML) have been recorded in patients following prolonged (5-10 years) exposure to alkylating agents (10-12).

TMZ is a relatively new drug and glioblastoma is an aggressive neoplasm with poor prognosis, thus the types of secondary cancer arising due to TMZ treatment have not yet been fully characterized. A review of the existing literature demonstrated that only 7 cases of TMZ-related acute lymphoblastic leukemia (ALL) have been reported thus far (13-18). The current case describes an 11-year-old boy with glioblastoma multiforme, who developed B-cell ALL 6 months after the last dose of TMZ. To the best of our knowledge, the present case is the youngest known patient with TMZ-related ALL. In addition, a review of the available literature regarding TMZ-related ALL was performed.

\section{Case report}

An 11-year-old boy presented at The First Affiliated Hospital of Jilin University (Changchun, China) in July 2013 with a 3-day history of generalized tonic-clonic seizures. The patient 
was otherwise healthy and had no other significant medical or family history. Magnetic resonance imaging (MRI) of the brain revealed a contrast-enhanced lesion $(4.5 \times 3 \mathrm{~cm})$ in the left temporo-occipital region with focal cystic degeneration (Fig. 1). Craniotomy and gross-total resection were subsequently performed. A post-operative pathological examination identified that the tumor cells varied in size, the nucleus was enlargened, the chromatin was granulated, and thickened-karyotheca and focal necrosis were observed. (Fig. 2; hematoxylin and eosin staining). Immunochemistry, performed as previously described (19) revealed positive staining for epidermal growth factor receptor (anti-EGFR; cat. no. SP111; dilution, 1:100; Fuzhou Maixin Biotech Co., Ltd., Fuzhou, China), sixty percent positive staining for Ki-67 (anti-Ki-67; cat. no. MX006; dilution, 1:100), GFAP (anti-GFAP; cat. no. GA-5; dilution, 1:100; both Fuzhou Maixin Biotech Co., Ltd.). Pathological analysis confirmed the diagnosis of giant cell glioblastoma with necrosis (Fig. 2) and this was classified as World Health Organization grade IV based on the 2007 World Health Organization Classification of Tumors of the Central Nervous System (20).

Postoperatively, the patient received TMZ-based concurrent chemoradiation with TMZ at a dose of $75 \mathrm{mg} / \mathrm{m}^{2} /$ day for 5 days during radiotherapy in August 2013. Radiotherapy was administered at a total dose of $60 \mathrm{~Gy}$ in 30 fractions for 6 weeks (5 days per week) and limited to the temporo-occipital region. Following concurrent chemoradiotherapy, the patient received a total of 5 cycles of maintenance TMZ from September 2013 to January $2014\left(150 \mathrm{mg} / \mathrm{m}^{2} /\right.$ day for 5 days every 28 day course). During this period the patient remained stable with no evidence of recurrence on surveillance MRI examinations.

At 6 months after the final dose of TMZ, the patient presented with increasing fatigue and easy bruising. Physical examination reported pallor, scattered petechiae and ecchymosis of the skin, tenderness of the sternum on percussion and hepatosplenomegaly (the liver was firm and palpable $6 \mathrm{~cm}$ below the costal margin). Blood counts indicated absolute lymphocytosis (white blood cell count, 16,750/ $\mu 1$, normal range, 3,500-9,500/ $\mu 1$, including 74\% lymphocytes, normal range $20-50 \%$ ) with anemia (hemoglobin count, $75 \mathrm{~g} / 1$, normal range $130-175 \mathrm{~g} / \mathrm{l}$ ) and thrombocytopenia (platelet count, $24,000 / \mu 1$; normal range, $125,000-350,000 / \mu 1)$. The serum lactate dehydrogenase (LDH) level was markedly elevated, at 1,243 U/1. Bone marrow aspiration exhibited $91.5 \%$ lymphocytes with $46 \%$ lymphoblasts (Fig. 3), and bone marrow biopsy demonstrated diffuse infiltration of the peripheral blood by lymphoblasts (Fig. 4). Peripheral blood flow cytometry indicated $63.58 \%$ phenoty pically abnormal cells, which were positive for CD10 (anti-CD10 mAb; cat. no. MX002; dilution, 1:100), CD19 (anti-CD19 mAb; cat. no. LE-CD19; dilution, 1:100), CD20 (anti-CD20 mAb; cat. no. MX003; dilution, 1:100), CD22 (anti-CD22 mAb; cat. no. MS-1087; dilution, 1:100) and CD79a (anti-CD79a mAb; cat. no. SP18; dilution, 1:100; all Fuzhou Maixin Biotech Co., Ltd.), and negative for immunoglobulin $\mathbf{M}$ (anti-Immunoglobulin $\mathrm{M}$; cat. no. 20-2786; dilution, 1:100; Dakewe Biotech Ltd., Shanghai, China) and CD7 (anti-CD7 mAb; cat. no. 272; dilution, 1:100; Fuzhou Maixin Biotech Co., Ltd.). Staining was performed as described previously (21). Bone marrow cytogenetics revealed a normal male karyotype (Fig. 5) and

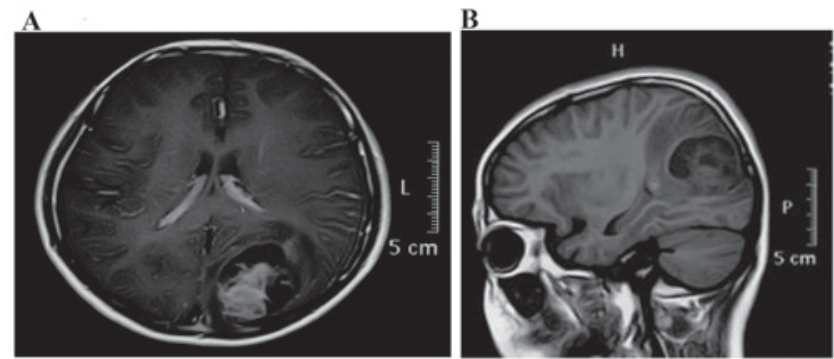

Figure 1. (A) Coronal and (B) sagittal T1-weighted MRI scans presenting a contrast-enhancing lesion $(4.5 \times 3 \mathrm{~cm})$ in the left temporo-occipital region with focal cystic degeneration. MRI, magnetic resonance imaging.

an MRI scan demonstrated no signs of recurrence. Based on these results, the patient was diagnosed with B-cell ALL. A four-week combination chemotherapy induction protocol was administered, comprising vincristine $\left(1.5 \mathrm{mg} / \mathrm{m}^{2}\right.$, intravenous injection at days 8, 15, 22 and 29), daunorubicin $\left(30 \mathrm{mg} / \mathrm{m}^{2}\right.$, intravenous injectionat days 8-10, 22 and 29), L-asparaginase (6,000-10,000 U/kg, intravenous injectionat days 11, 13, 15, 17, $19,21,23,25,27$ and 29) and prednisone $\left(60 \mathrm{mg} / \mathrm{m}^{2} /\right.$ day, oral administration in days $1-7 ; 40 \mathrm{mg} / \mathrm{m}^{2} /$ day, oral administration in days 8-28), and the patient achieved complete hematological remission. The patient remains on chemotherapy and has been in remission for 8 months, undergoing regular surveillance MRI three times a month.

\section{Discussion}

A large number of randomized clinical trials have demonstratedthe safety and efficacy of TMZ in the treatment of aggressive central nervous system tumors, revealing significantly improved overall survivalcompared with the first-generation oral alkylating agent (6-8). The United States Food and Drug Administration approved TMZ as a treatment for glioblastoma in 1999 (22). However, TMZ is a relatively new alkylating agent and its long-term safety is not fully characterized.

The present study performed a literature review and identified only 6 cases of therapy-associated ALL that occurred subsequent to TMZ administration include the current patient. The clinical profiles of the six patients are summarized in Table I. Of the patients identified, three were males and three werefemales and all except onewere adults. A total of six patients were diagnosed with primary glioblastoma and received TMZ-based concurrent chemoradiation. The other patient was diagnosed with oligodendroglioma and was treated with TMZ-based adjuvant chemotherapy. Bone marrow cytogenetics revealed chromosome abnormalities in 2 cases: 1 with $\mathrm{t}(4 ; 11)(\mathrm{q} 21 ; \mathrm{q} 23)(14)$, and the other with breakpoint cluster region/Abelson murine leukemia rearrangement, $t(9 ; 22)$ and monosomy 7 (13). During concurrent chemoradiation, TMZ was administered orally at $75 \mathrm{mg} / \mathrm{m}^{2} / \mathrm{d} 5$ consecutive days a week for 42 days. Subsequent maintenance therapy with TMZ was administered at $150 \mathrm{mg} / \mathrm{m}^{2} /$ day for 5 days in a 28 day cycle, with the total number of cycles ranging from 1-36 prior to the diagnosis of secondary hematological disorders. A total of 5 patients were diagnosed with pre-B ALL, and the other two with precursor T-cell ALL (pre-T ALL). 
A

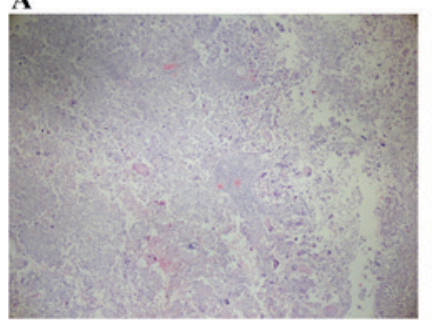

B

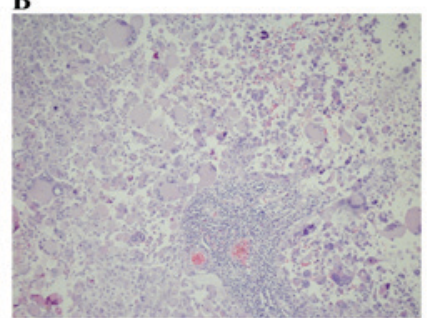

Figure 2. Histopathological examination at magnifications of (A) $\mathrm{x} 40$ and (B) $\mathrm{x} 1,000$ confirmed diagnosis of giant cell glioblastoma with necrosis at World Health Organization grade IV (staining, hemaotoxylin and eosin).
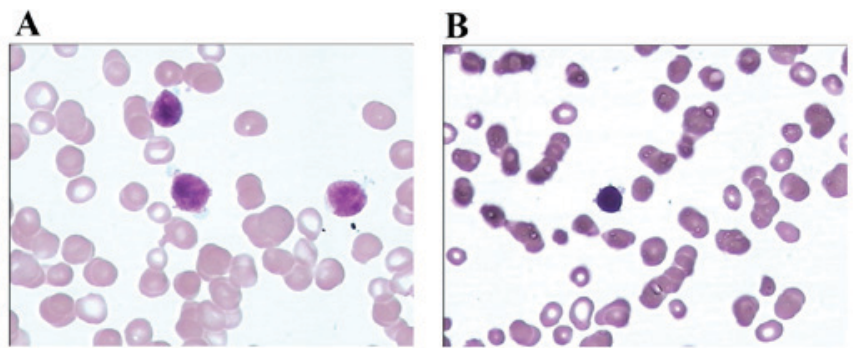

Figure 3. Bone-marrow smear examination at magnifications of (A) $x 1,000$ and (B) $x 40$ revealed lymphocytosis $(91.5 \%)$, in which lymphoblasts and immature lymphocytes constitute $46 \%$ of all lymphocytes (staining, Wright-Giemsa). This confirmed a diagnosis of acute lymphoblastic leukemia.
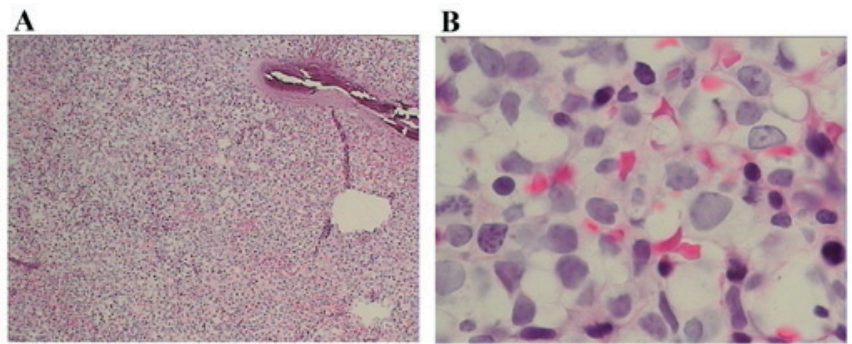

Figure 4. Bone-marrow biopsy examination at magnification of (A) x1,000 and (B) x400 demonstrated diffuse infiltration with lymphoblasts (staining, Wright-Giemsa).

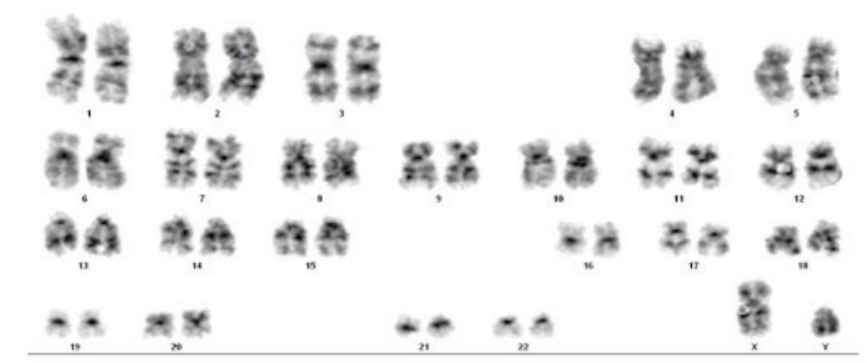

Figure 5. Bone marrow cytogenetics (G-banding), presenting a normal karyotype.

The leukemogenic potential of alkylating agents is well-known and it usually takes 5-10 years after exposure for leukemia to develop (10-12). Following prolonged TMZ treatment, t-MDS and t-AML have been widely reported in patients (22-24). Results from phase I and II clinical trials suggest that the primary toxicity associated with TMZ is myelotoxicity (13). Reported TMZ-related secondary hematological disorders, including t-MDS and t-AML, are usually secondary to the administration of TMZ in combination with other alkylating agents (25) and few cases have been documented following TMZ monotherapy $(24,26)$. Meanwhile, T-ALL subsequent to TMZ-based concurrent chemoradiation in patients with glioblastoma is extremely rare (13-18). In the limited number of patients studied, B-ALL was more common than T-ALL.

The first case of TMZ therapy-related ALL was reported by De Vita et al (13) in 2005, documenting a case of $\mathrm{Ph}+$ acute precursor B-cell lymphoblastic leukemia French-American-British L1 subtype. Subsequently, four other cases were reported with B- or T-ALL with or without cytogenetic abnormalities (14-17). The latency period from the first dose of TMZ to the onset of ALL was an average of 17.7 months (range, 4-57 months), which is notably shorter than the latency period of therapy-related ALL subsequent to other conventional alkylating agents (mean, 63 months) (27). The development of secondary malignancies usually requires the accumulation of multiple genetic abnormalities, causing a period of latency.

Treatment-related MDS and t-AML are the most common types of secondary leukemia and secondary ALL accounts for $\sim 10 \%$ of all secondary leukemia cases $(28,29)$. TMZ-related ALL is rare and the specific mechanisms underlying its development remain unclear. Previous studies suggest that several genetic predisposing factors may be involved in the pathogenesis of secondary ALL. De Vita et al (13) indicated that monosomy 7 and $t(9 ; 22)$ may be associated with the onset of TMZ-related ALL. In addition, Chou et al (14) suggested that $\mathrm{t}(4 ; 11)(\mathrm{q} 21 ; \mathrm{q} 23)$ may be a potential genetic predisposing factor (14). However, the literature review performed in the current study demonstrated that $>50 \%$ of the secondary ALL cases exhibited no associated chromosome abnormality (15-17). Onset of ALL may thus be an incidental event following TMZ treatment, and the relatively short latency period suggests that TMZ administration may be the primary pathogenic factor contributing to the development of TMZ-related ALL. Geiger et al (30) confirmed the mutagenic potential of TMZ for bone marrow cells in an in vivo murine model and suggested that this may be the underlying cause of therapy-related leukemia in TMZ-treated patients.

The optimal treatment and prognosis of TMZ-related ALL remain undetermined. To the best of our knowledge, the present case is only the sixth patient reported with TMZ-related ALL, and also the youngest. Half of all patients have achieved complete remission following induction chemotherapy. However, the therapy details and outcomes of these cases are incomplete. In the present case, a standard combination chemotherapy protocol comprising vincristine, daunorubicin, L-asparaginase and prednisone was employed and the outcome was favorable. The implication of therapy-related leukemia is that the prognosis may be worse than de novo leukemia; however, it has been consistently demonstrated in various studies that standard or more intensive therapies improve patient outcomes (31-33).

A high index of suspicion is required by practitioners when following up patients with glioblastoma who have received 


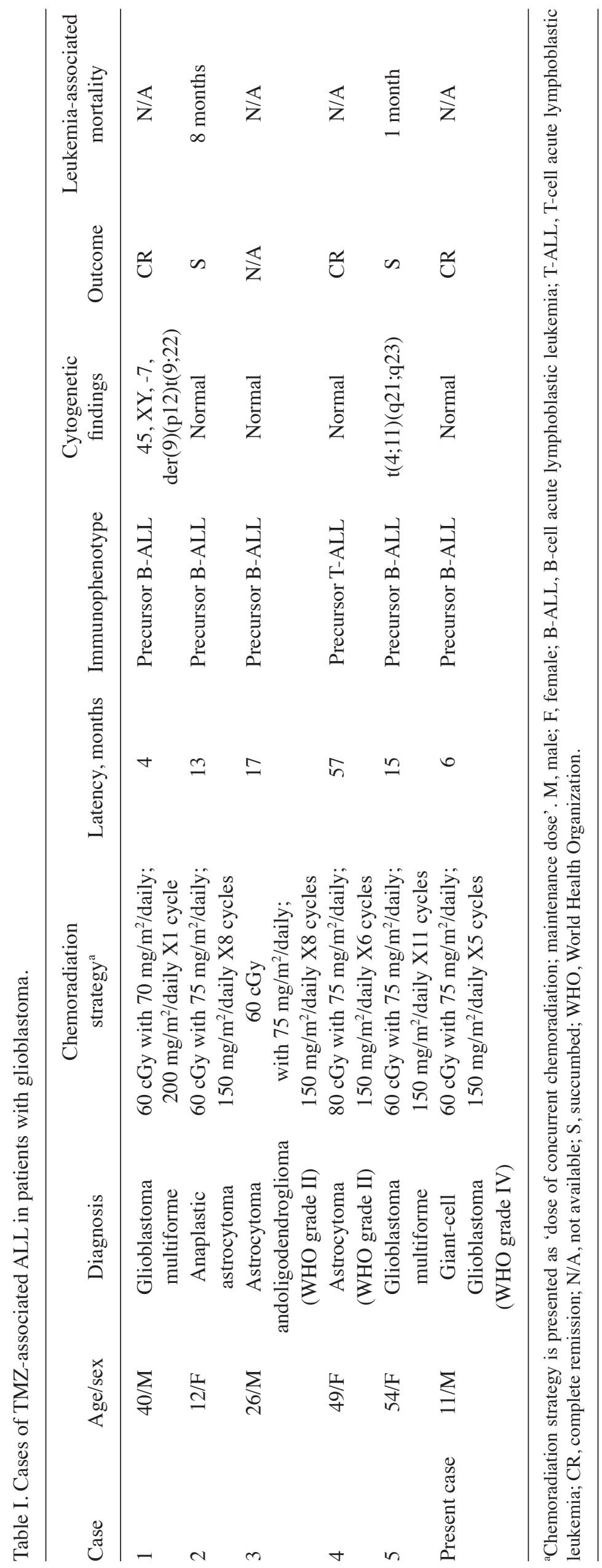


TMZ as a part of their treatment. Perry et al (34) suggested that the cumulative dose of alkylating agents is a major risk factor for the pathogenesis of secondary leukemia, and concurrent radiotherapy does not appear to confer additional leukemogenic risk. Pagano et al $(28,35)$ supported this hypothesis and added that it is not only exposure to chemotherapy, but also genetic predisposition that is important in the pathogenesis of secondary leukemia.

Alkylating agents have been used to treat high-grade gliomas for decades and their leukemogenicity is well known. However, TMZ is a second-generation oral alkylating agent and has only been widely used for the last decade, therefore its leukemogenicity has not been comprehensively evaluated. TMZ-related hematological disorders have increasingly been reported in the literature, suggesting that TMZ has a similar leukemogenic potential to other alkylating agents $(9,25)$. However, the latency period from TMZ exposure to the development of secondary leukemia appears to be considerably shorter than other alkylating agents.

In conclusion, $\mathrm{TMZ}$ is included in the standard treatment of high-grade gliomas and it is generally safe and effective; however, its leukemogenic potential should be noted. Close hematological monitoring and a high index of suspicion by practitioners is required, and further studies are warranted to determine the specific pathogenic mechanism of TMZ-related ALL.

\section{Acknowledgements}

The authors would like to thank Dr Jinlu Yu (The First Hospital of Jilin University, Changchun, Jilin, China) for his contribution to the language editing of the present manuscript.

\section{Funding}

No funding was received.

\section{Availability of data and materials}

The datasets used and/or analyzed during the current study are available from the corresponding author on reasonable request.

\section{Authors' contributions}

HH conceived and designed the study. PLi, TL and LQ acquired the data, acquired and managed the patients and provided the radiology images. QM contributed to the study design and PL analyzed and interpreted the data. HH supervised the study.

\section{Ethics approval and consent to participate}

The present study was approved by the Ethics Committee of the First Hospital of Jilin University. The patient provided written informed consent for the present study.

\section{Consent for publication}

The patient and his father consented to contribute his radiology images, hematology and pathological sections to medical research, for copyright and ethics without controversy.

\section{Competing interests}

The authors declare that there are no competing interests.

\section{References}

1. Macdonald DR: Temozolomide for recurrent high-grade glioma. Semin Oncol 28 (4 Suppl 13): S3-S12, 2001.

2. Yung WK: Temozolomide in malignant gliomas. Semin Oncol 27 (3 Suppl 6): S27-S34, 2000

3. Wang T, Pickard AJ and Gallo JM: Histone methylation by temozolomide; a classic DNA methylating anticancer drug. Anticancer Res 36: 3289-3299, 2016.

4. Roos WP, Batista LF, Naumann SC, Wick W, Weller M, Menck CF and Kaina B: Apoptosis in malignant glioma cells triggered by the temozolomide-induced DNA lesion O6-methylguanine. Oncogene 26: 186-197, 2007.

5. Villano JL, Seery TE and Bressler LR: Temozolomide in malignant gliomas: Current use and future targets. Cancer Chemother Pharmacol 64: 647-655, 2009.

6. Le Rhun E, Taillibert S and Chamberlain MC: Anaplastic glioma: Current treatment and management. Expert Rev Neurother 15: 601-620, 2015.

7. Nayak L, Panageas KS, Reiner AS, Huse JT, Pentsova E, Braunthal SG, Abrey LE, DeAngelis LM and Lassman AB: Radiotherapy and temozolomide for anaplastic astrocytic gliomas. J Neurooncol 123: 129-134, 2015.

8. Taal W, Bromberg JE and van den Bent MJ: Chemotherapy in glioma. CNS Oncol 4: 179-192, 2015.

9. Momota H, Narita Y, Miyakita Y and Shibui S: Secondary hematological malignancies associated with temozolomide in patients with glioma. Neuro Oncol 15: 1445-1450, 2013.

10. Björkholm M, Hultcrantz M and Derolf $\AA$ R: Leukemic transformation in myeloproliferative neoplasms: Therapy-related or unrelated. Best Pract Res Clin Haematol 27: 141-153, 2014.

11. Ishikawa M, Nakayama K, Rahman MT, Rahman M, Katagiri H, Katagiri A, Ishibashi T, Iida K, Nakayama $\mathrm{N}$ and Miyazaki K: Therapy-related myelodysplastic syndrome and acute myeloid leukemia following chemotherapy (paclitaxel and carboplatin) and radiation therapy in ovarian cancer: A case report. Eur J Gynaecol Oncol 35: 443-448, 2014.

12. Hamano A, Shingaki S, Abe Y, Miyazaki K, Sekine R, Nakagawa Y, Tsukada N, Hattori Y and Suzuki K: Impacts of new agents for multiple myeloma on development of secondary myelodysplastic syndrome and acute myeloid leukemia. Rinsho Ketsueki 55: 428-435, 2014 (In Japanese).

13. De Vita S, De Matteis S, Laurenti L, Chiusolo P, Reddiconto G, Fiorini A, Leone G and Sica S: Secondary Ph+ acute lymphoblastic leukemia after temozolomide. Ann Hematol 84: 760-762, 2005.

14. Chou KN, Lin YC, Liu MY and Chang PY: Temozolomide-related acute lymphoblastic leukemia with translocation $(4 ; 11)(\mathrm{q} 21 ; \mathrm{q} 23)$ in a glioblastoma patient. J Clin Neurosci 21: 701-704, 2014.

15. Shaikh AJ and Masood N: Acute lymphoblastic leukemia subsequent to temozolomide use in a 26-year-old man: A case report. J Med Case Rep 4: 274, 2010.

16. Ogura M, Todo T, Tanaka M, Nannya Y, Ichikawa M, Nakamura F and Kurokawa M: Temozolomide may induce therapy-related acute lymphoblastic leukaemia. Br J Haematol 154: 663-665, 2011.

17. Momota H, Narita Y, Miyakita Y, Hosono A, Makimoto A and Shibui S: Acute lymphoblastic leukemia after temozolomide treatment for anaplastic astrocytoma in a child with a germline TP53 mutation. Pediatr Blood Cancer 55: 577-579, 2010.

18. Prevost C, Ferrandi D, Corsetti MT, Mascolo M, Depaoli L, Salvi F, Bottaro R, Melato M, Palermo M and Ruiz L: p17.72acute lymphoblastic leukemia after temozolomide treatment for oligodendroglioma: Case report. Neuro Oncol 16 (Suppl 2): ii104, 2014.

19. Kiviniemi A, Gardberg M, Frantzén J, Parkkola R, Vuorinen V, Pesola M and Minn H: Serum levels of GFAP and EGFR in primary and recurrent high-grade gliomas: Correlation to tumor volume, molecular markers, and progression-free survival. J Neurooncol 124: 237-245, 2015.

20. Louis DN, Ohgaki H, Wiestler OD, Cavenee WK, Burger PC, Jouvet A, Scheithauer BW and Kleihues P: The 2007 WHO classification of tumours of the central nervous system. Acta Neuropathol 114: 97-109, 2007. 
21. Alvarnas JC, Brown PA, Aoun P, Ballen KK, Barta SK, Borate U, Boyer MW, Burke PW, Cassaday R, Castro JE, et al: acute lymphoblastic leukemia, Version 2.2015. J Natl Compr Canc Netw 13: 1240-1279, 2015.

22. Villano JL, Letarte N, Yu JM, Abdur S and Bressler LR: Hematologic adverse events associated with temozolomide. Cancer Chemother Pharmacol 69: 107-113, 2012.

23. Scaringi C, De Sanctis V, Minniti G and Enrici RM: Temozolomide-related hematologic toxicity. Onkologie 36 444-449, 2013

24. Su YW, Chang MC, Chiang MF and Hsieh RK: Treatment-related myelodysplastic syndrome after temozolomide for recurrent high-grade glioma. J Neurooncol 71: 315-318, 2005.

25. Kim SJ, Park TS, Lee ST, Song J, Suh B, Kim SH, Jang SJ, Lee CH and Choi JR: Therapy-related myelodysplastic syndrome/acute myeloid leukemia after treatment with temozolomide in a patient with glioblastoma multiforme. Ann Clin Lab Sci 39: 392-398, 2009.

26. Chamberlain MC and Raizer J: Extended exposure to alkylator chemotherapy: Delayed appearance of myelodysplasia. J Neurooncol 93: 229-232, 2009.

27. Mauritzson N, Albin M, Rylander L, Billström R, Ahlgren T, Mikoczy Z, Björk J, Strömberg U, Nilsson PG, Mitelman F, et al: Pooled analysis of clinical and cytogenetic features in treatment-related and de novo adult acute myeloid leukemia and myelodysplastic syndromes based on a consecutive series of 761 patients analyzed 1976-1993 and on 5098 unselected cases reported in the literature 1974-2001. Leukemia 16: 2366-2378, 2002.

28. Pagano L, Pulsoni A, Tosti ME, Annino L, Mele A, Camera A, Martino B, Guglielmi C, Cerri R, Di BE, et al: Acute lymphoblastic leukaemia occurring as second malignancy: Report of the GIMEMA archive of adult acute leukaemia. Gruppo Italiano Malattie Ematologiche Maligne dell' Adulto. Br J Haematol 106: 1037-1040, 1999.
29. Hunger SP, Sklar J and Link MP: Acute lymphoblastic leukemia occurring as a second malignant neoplasm in childhood: Report of three cases and review of the literature. J Clin Oncol 10: 156-163, 1992.

30. Geiger H, Schleimer D, Nattamai KJ, Dannenmann SR, Davies SM and Weiss BD: Mutagenic potential of temozolomide in bone marrow cells in vivo. Blood 107: 3010-3011, 2006.

31. Alam N, Atenafu EG, Kuruvilla J, Uhm J, Lipton JH, Messner HA, Kim DH, Seftel M and Gupta V: Outcomes of patients with therapy-related AML/myelodysplastic syndrome (t-AML/MDS) following hematopoietic cell transplantation. Bone Marrow Transplant 50: 1180-1186, 2015.

32. Aldoss I, Dagis A, Palmer J, Forman S and Pullarkat V: Therapy-related ALL: Cytogenetic features and hematopoietic cell transplantation outcome. Bone Marrow Transplant 50: 746-748, 2015.

33. Ferrero D, Crisà E, Marmont F, Audisio E, Frairia C, Giai V, Gatti T, Festuccia M, Bruno B, Riera L, et al: Survival improvement of poor-prognosis AML/MDS patients by maintenance treatment with low-dose chemotherapy and differentiating agents. Ann Hematol 93: 1391-1400, 2014.

34. Perry JR, Brown MT and Gockerman JP: Acute leukemia following treatment of malignant glioma. J Neurooncol 40: 39-46, 1998.

35. Pagano L, Pulsoni A, Tosti ME, Avvisati G, Mele L, Mele M, Martino B, Visani G, Cerri R, Di Bona E, et al: Clinical and biological features of acute myeloid leukaemia occurring as second malignancy: GIMEMA archive of adult acute leukaemia. Br J Haematol 112: 109-117, 2001. 